\title{
Research Article \\ Benefit of the NeQuick Galileo Version in GNSS Single-Point Positioning
}

\author{
Antonio Angrisano, Salvatore Gaglione, Ciro Gioia, Marco Massaro, and Salvatore Troisi \\ Department of Science and Technology, PArthenope Navigation Group (PANG), Parthenope University of Naples, \\ Centro Direzionale di Napoli Isola C4, 80143 Naples, Italy
}

Correspondence should be addressed to Salvatore Gaglione; salvatore.gaglione@uniparthenope.it

Received 31 May 2013; Revised 17 September 2013; Accepted 30 September 2013

Academic Editor: Yuei-An Liou

Copyright (C) 2013 Antonio Angrisano et al. This is an open access article distributed under the Creative Commons Attribution License, which permits unrestricted use, distribution, and reproduction in any medium, provided the original work is properly cited.

\begin{abstract}
The GNSS measurements are strongly affected by ionospheric effects, due to the signal propagation through ionosphere; these effects could severely degrade the position; hence, a model to limit or remove the ionospheric error is necessary. The use of several techniques (DGPS, SBAS, and GBAS) reduces the ionospheric effect, but implies the use of expensive devices and/or complex architectures necessary to meet strong requirements in terms of accuracy and reliability for safety critical application. The cheapest and most widespread GNSS devices are single frequency stand-alone receivers able to partially correct this kind of error using suitable models. These algorithms compute the ionospheric delay starting from ionospheric model, which uses parameters broadcast within the navigation messages. NeQuick is a three-dimensional and time-dependent ionospheric model adopted by Galileo, the European GNSS, and developed by International Centre for Theoretical Physics (ICTP) together with Institute for Geophysics, Astrophysics, and Meteorology of the University of Graz. The aim of this paper is the performance assessment in single point positioning of the NeQuick Galileo version provided by ESA and the comparison with respect to the Klobuchar model used for GPS; the analysis is performed in position domain and the errors are examined in terms of RMS and maximum error for the horizontal and vertical components. A deep analysis is also provided for the application of the exanimated model in the first possible Galileo only position fix.
\end{abstract}

\section{Introduction}

Global Navigation Satellite System (GNSS) provides, with global coverage and in all weather conditions, threedimensional coordinates, velocity, and time synchronization for users equipped with a receiver/processor [1]. The accuracy of GNSS depends on observables accuracy, satellite geometry, number of tracked satellites, and operational scenario. Table 1 quantifies the main errors affecting the observable accuracy.

The ionosphere is the main error source in GNSS measurements. At times, the range error of the troposphere and the ionosphere can be comparable, but the variability of Earth's ionosphere is much larger and more difficult to model.

The range of the ionospheric error can vary from a few meters to 30 meters at the zenith, depending on observation epoch and latitude [2], whereas the tropospheric range error at the zenith is generally between two to three meters.
Although the range error of the troposphere generally does not change by more than $\pm 10 \%$, even for long periods of time, the ionosphere frequently changes by at least one-order of magnitude during the course of each day [3].

The ionosphere is the ionized region of the upper atmosphere, which extends from about $50 \mathrm{~km}$ to more than $1000 \mathrm{~km}$ where the density of free electrons and ions significantly influences the propagation of electromagnetic radio frequency waves [4]. The structure and the properties of the ionosphere depend essentially on solar activity, variations of Earth's magnetic field (geomagnetic field effect), movements of neutral "wind" in the upper atmosphere due to Earth's rotation, the effects of electrical current and ambient electrical fields, the density and the content of the atmosphere at different altitudes and geographical latitudes, and so on. Usually, the ionosphere is separated into five independent regions, sometimes called layers as depicted in Figure $1[5,6]$. 
TABLE 1: Statistical ranging error budget for GNSS single-frequency receiver [2].

\begin{tabular}{lc}
\hline Error source & $1 \sigma$ error $(\mathrm{m})$ \\
\hline Ephemeris data & 2.1 \\
Satellite clock & 2.1 \\
Ionosphere & 4.0 \\
Troposphere & 0.7 \\
Multipath & 1.4 \\
Receiver noise & 0.5 \\
\hline User equivalent range error & 5.3 \\
\hline
\end{tabular}

The bottom one, from 50 to $80 \mathrm{~km}$, is called the D layer; from 80 to $130 \mathrm{~km}$ is the E layer and above $150 \mathrm{~km}$ is the F layer. The latter region usually consists of the F1 layer, from 130 to 200$250 \mathrm{~km}$, and in the F2 layer, above $250 \mathrm{~km}$. Apart from these layers, at $90-120 \mathrm{~km}$, the sporadic layer Es is observed, whose vertical thickness is small; its occurrence is usually explained by the influence of a neutral wind of atmospheric gases on the charged particles of plasma, resulting in exchange of plasma accompanied by stratified wind along the height [7]. The corpuscular solar radiation penetrates the ionosphere mainly at the high latitudes, called the polar ionosphere, whereas in the middle and lower latitude ionosphere, the properties of plasma are determined by solar wave radiation, mostly for the D layer, because the ionization occurs here only in the diurnal periods $[8,9]$.

Different methods can be adopted to minimize the ionospheric effect, such as the use of

(i) dual-frequency technique,

(ii) augmentation system,

(iii) ionospheric model.

The ionosphere refractive index depends on the operating frequency so a dual-frequency GNSS user can take advantage of this feature and correct the measurements with an estimation of ionospheric delay obtained by a linear combination of dual frequency measures (this technique is referred to as iono-free). This method is the most effective but it cannot be used in a single frequency receiver [10].

Alternatively, GNSS receivers can obtain the ionospheric correction through augmentation systems (such as Differential GPS-DGPS, or Satellite-Based Augmentation SystemSBAS), based on differential corrections, computed by a single station or by a network, and broadcasted to the receivers via terrestrial or satellite radio link.

Augmentation systems involve the use of complex architectures, while the algorithms using broadcast ionospheric parameters, within navigation messages, are cheaper and easy to be implemented in commercial single frequency receivers. Klobuchar developed the first algorithm for ionospheric correction in the mid-1970s for the GPS single frequency user, able to correct approximately $50 \%$ of the ionospheric range error [11]. The $50 \%$ correction goal was established because the GPS satellite message had space for only eight coefficients

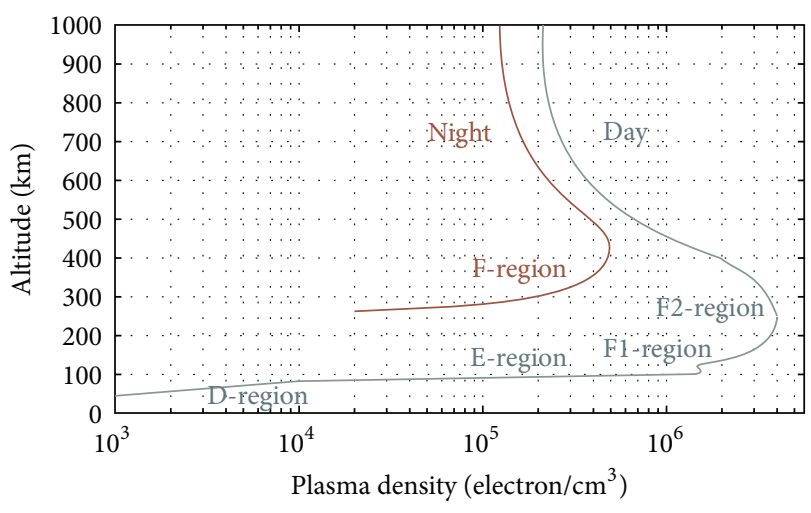

FIGURE 1: Ionospheric profile and ionospheric regions.

to describe the worldwide behavior of the Earth's ionosphere. This choice represents also a trade-off between computational complexity and precision to be achieved with this kind of models [3].

NeQuick is an ionospheric model developed at the Aeronomy and Radiopropagation Laboratory of the Abdus Salam International Centre for Theoretical Physics (ICTP) Trieste, Italy, and at the Institute for Geophysics, Astrophysics and Meteorology of the University of Graz, Austria. It has been considered as an evolution of the DGR profiler proposed by Di Giovanni and Radicella [12-14], subsequently modified by Radicella and Zhang [15]. Three versions of NeQuick model currently exist. NeQuick 1 is the original version, adopted by ITU-R (International Telecommunication Union-Radiocommunication Sector) [12]. The original version has been improved, generating two subsequent versions: NeQuick Galileo version (NeQuick G), developed by ESA (European Space Agency) and considered for the final Galileo receiver algorithm, and the latest $\mathrm{NeQuick} 2$, an update of the first analytical model formulation that takes advantage of the increasing amount of available data and provides a better representation of the average ionosphere behavior at global scale.

The remainder of this paper is organized as follows. The Position Velocity and Time (PVT) algorithm is described in Section 2. A brief review on examined ionospheric models is provided in Section 3, highlighting the updating of the previous NeQuick model. NeQuick Galileo version and Klobuchar models are compared in terms of achieved positioning accuracy in Section 4. In Section 5 a positioning using only Galileo measurements is carried out using the considered ionospheric models. Some conclusions are finally discussed in Section 6.

\section{PVT Algorithm}

GNSS positioning is based on the one-way ranging technique: the time of travel of a signal, transmitted by a satellite, is 
measured and scaled by the speed of light to obtain the pseudorange $(\mathrm{PR})$ as follows:

$$
\rho=d+c \delta t_{u}+c \delta t_{s}+d_{T}+d_{I}+\varepsilon_{\rho},
$$

where $\rho$ is the PR measurement, $d$ is the geometric satellitereceiver distance, $c \delta t_{u}$ is the receiver clock offset scaled by the speed of light $c, c \delta t_{s}$ is the satellite clock offset scaled by speed of light, $d_{T}$ is the tropospheric delay, $d_{I}$ is the ionospheric delay, and $\varepsilon_{\rho}$ contains the other error sources.

Using trilateration technique it is possible to obtain the navigation solution composed by the tridimensional receiver coordinates and the receiver clock offset relative to system time scale.

The measurement equations (1) are not linear for the unknowns then they have to be linearized, obtaining

$$
\underline{\Delta \rho}=H * \underline{\Delta x}+\underline{\varepsilon}
$$

where $\Delta \rho$ is the vector containing the difference between actual $\overline{\text { and }}$ predicted PR measurements, $H$ is the design matrix, $\underline{\varepsilon}$ is the residual error vector, and $\underline{\Delta x}$ is the state vector defined as:

$$
\underline{\Delta x}=\left[\underline{\Delta P} \Delta\left(c \delta t_{u}\right)\right] .
$$

A least squares method is used to solve (3) as

$$
\underline{\Delta x}=\left(H^{T} W H\right)^{-1} H^{T} W \underline{\Delta \rho},
$$

where $W$ is a weighting matrix based on a satellite elevation dependent measurement model [16].

In this work GPS single point position is carried out and the $\mathrm{PR}$ are corrected for the satellite related error, tropospheric effect using Hopfield model, relativistic and Sagnac effects; the ionospheric delay is computed using Klobuchar and NeQuick G model, in order to compare their performance.

\section{Klobuchar and NeQuick Ionospheric Models}

Single frequency users have to correct as much as possible the first-order ionospheric term, which accounts for more than 99.9\% of the total ionospheric delays [17]. All the Ionospheric Correction Algorithms (ICA), providing ionospheric delay estimation, start from the evaluation of the electron concentration $\left(N_{e}\right)$ relative to the path from satellite to user. The firstorder ionospheric delay is related to the signal frequency $f$ and to the Slant Total Electron Content (STEC), defined as the electron concentration along the path between the receiver $\mathrm{R}$ and satellite $S$, and is shown as

$$
\mathrm{STEC}=\int_{\mathrm{R}}^{\mathrm{S}} N_{e} \mathrm{~d} l .
$$

The dispersive effect of the ionosphere on GNSS observables causes a code delay obtained from

$$
d_{I}=\frac{40.3 \cdot \mathrm{STEC}}{f^{2}}
$$

where $d_{I}$ is expressed in meters, STEC in TECu (TEC units; $1 \mathrm{TECu}=10^{16} \mathrm{el} / \mathrm{m}^{2}$ ), and $f$ in $\mathrm{MHz}$. The value of $1 \mathrm{TECu}$ for the $\mathrm{L} 1$ frequency $(1575.42 \mathrm{MHz})$ generates a delay of $0.16 \mathrm{~m}$.

Below the official ICA for GPS (Klobuchar) and for Galileo (NeQuick G) are described.

3.1. Klobuchar Model. Ionospheric Klobuchar model is an algorithm developed, around the mid-70s by Klobuchar, for single-frequency GPS receivers to correct approximately $50 \%$ of ionospheric delay [11] and was designed based on the Bent model [18]. It is defined as a single layer ionospheric model (SLM-Single Layer Model), because the ionosphere (i.e., its TEC) is assumed as concentrated in an infinitesimal layer, placed at an average altitude of $350 \mathrm{~km}$ from the Earth's surface.

In a SLM the STEC is calculated at the Ionospheric Point (IP) which is a geographic point, obtained by the intersection between the propagation direction (ray path, also called Line Of Sight-LOS) and the average height of the ionosphere. The projection on the surface of the ionospheric point is the Subionospheric Point (SIP) depicted in Figure 2 [10].

Klobuchar model provides a different estimation for the daytime and nighttime ionospheric delay (in seconds) along the SIP vertical direction, starting from eight coefficients (within GPS navigation message), which describe the worldwide ionosphere behavior [11]. Night time correction is assumed equal to a globally constant value of $5 \mathrm{~ns}$ $(\sim 1.5 \mathrm{~m})$ for L1 carrier, while the diurnal vertical delay is modeled as cosine featured by amplitude $A$, period $P$, and phase $\Phi$ (constant and equal to 14 hours) depending on the geomagnetic latitude of SIP. In order to compute $A$ and $P$ in any Earth position, eight coefficients (four for $A$ and four for $P$ ) of two third-degree polynomials, are broadcast daily in the GPS navigation message. The GPS Ground Control Segment updates these coefficients according to season and solar activity level.

3.2. NeQuick Model. NeQuick is a semiempirical model that describes spatial and temporal variations of the ionospheric electron density. It is based on the DGR ionospheric profiler [12] and provides both Vertical TEC (VTEC) and STEC for any specified path $[13,14]$.

NeQuick model uses the peaks of the three main ionospheric regions (E, F1, and F2) as anchor points $[14,19]$. The electron density at any location is computed starting from the characteristic parameters such as peak electron density and peak height; STEC is computed by integrating the electron density along the signal path. NeQuick model is at basis of the real-time ionospheric correction model algorithm used for Galileo single-frequency positioning [20]. A common ionospheric profile, with its main ionospheric regions for night and day time, is shown in Figure 1.

In the NeQuick model, the electron density above $100 \mathrm{~km}$ and up to the F2-layer peak (the bottom side region) is calculated by a modified DGR profile formulation which includes five semi-Epstein layers [21] with modeled thickness parameters [19]. In the topside (the region of the ionosphere above the F2-layer peak) the electron density is described by 


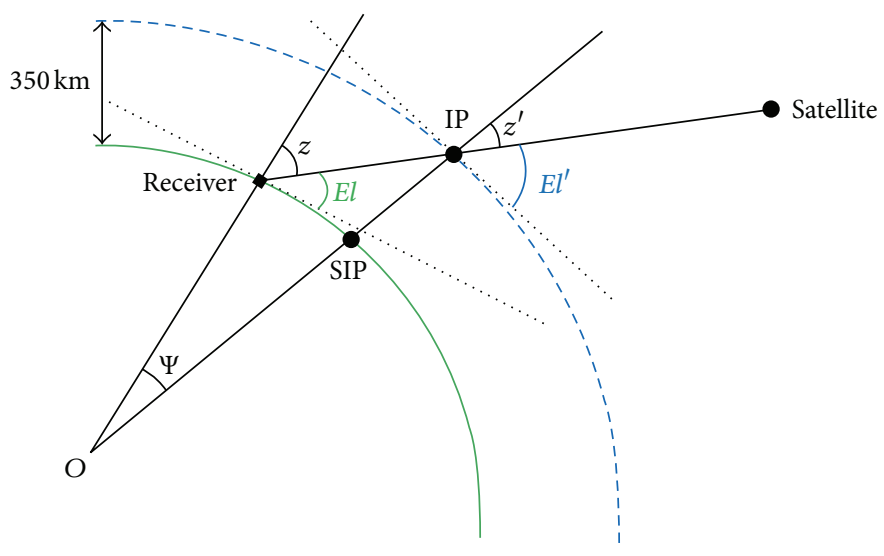

FIGURE 2: Klobuchar SLM.

only a semi-Epstein layer with a height-dependent thickness parameter [14] empirically determined [21, 22].

Taking advantage of the increasing amount of available data, the NeQuick model has been continuously updated with changes involving the formulation of some specific parameters although conceptual structure of the model remains unaltered $[13,14,19,23]$.

NeQuick 1 constitutes the original version recommended by the ITU-R. At first, it has been improved regarding the bottom side [19]: unrealistic electron density horizontal gradients at low and high latitudes, due to problematic transitions between layers, has been overcome reformulating the parameters associated with the E and F1 layers. These improvements have been included in two subsequent versions, the one currently considered for the final Galileo algorithm-NeQuick G-and the latest NeQuick 2 [24, 25]. NeQuick 2 model is characterized by a new formulation of the topside representation; specifically this change was obtain from topside soundings data from the ISIS-2 satellite (International Satellites for Ionospheric Studies) [22]. It provides electron density topside profiles closer to experimental ones as these profiles are usually underestimated by NeQuick 1 at high and midlatitudes and slightly overestimated at low latitudes. It is not possible to provide additional details about NeQuick G model because it has not yet been officially released by ESA.

NeQuick algorithm was originally developed to be used with monthly average solar flux index F10.7 (solar radio flux per unit frequency at a wavelength of $10.7 \mathrm{~cm}$ ); so in order to use NeQuick model in real time application such as GNSS ionospheric correction computation, the monthly average F10.7 index has to be replaced by a daily input parameter in order to take in account both daily variation of the solar activity and the user's local geomagnetic condition. This daily input parameter is the so-called effective ionization level $(A z)$ expressed in solar flux unit $\left(\mathrm{sfu}-10^{-22}(\mathrm{~W})\left(\mathrm{m}^{-2}\right)\left(\mathrm{Hz}^{-1}\right)\right)[20$, 26]. Thus, $A z$ plays the role of the solar activity information provided to the model in order to fit a specific dataset [27]. For Galileo single frequency operation, daily $A z$ values will be computed by ground segment using STEC measurements obtained during the previous 24 hours. From the calculated
$A z$ at different station of ground segment, the worldwide behaviour of $A z$ parameter is modeled by a second-order polynomial [20] as a function of receiver location as follows:

$$
A z(\mu)=a_{0}+a_{1} * \mu+a_{2} * \mu^{2},
$$

where $a_{0}, a_{1}$, and $a_{2}$ are coefficients created by optimizing the NeQuick model to a set of global network of permanent stations. Such coefficients are broadcast to the users within the Galileo navigation message [27] and updated once a day allowing them to run the model $[20,26] . \mu$ is Modified Dip latitude (MODIP), whose expression is shown below, Dip is the magnetic inclination [28]

$$
\mu=\tan ^{-1}\left(\frac{I}{\sqrt{\cos \phi}}\right)
$$

where $\phi$ is the geodetic latitude and $I$ the magnetic field inclination defined as the angle between the observer horizontal plane in the receiver position and the geomagnetic field direction.

Since the coefficients $a_{0}, a_{1}$, and $a_{2}$ are currently not included in the Galileo navigation message, in this research the parameter $A z$ has been preliminarily calculated for a series of worldwide distributed monitor stations with Brent method optimization [28-30]. Hence, the ionization level, relative to a defined station and day, has to minimize the sequence

$$
A z=\arg \min \sum_{i=1}^{n}\left|\operatorname{VTEC}_{\text {Reference }}-\operatorname{VTEC}_{\text {NeQuick }}(A z)\right|_{i}^{2},
$$

where $\mathrm{VTEC}_{\text {Reference }}$ is VTEC of reference for the station and is computed from the 1-day predicted Global Ionospheric Map (GIM) with a 2-hour resolution provided by Center for Orbit Determination in Europe (CODEhttp://www.aiub.unibe.ch/) in IONosphere EXchange (IONEX) format [31], $\operatorname{VTEC}_{\mathrm{NeQuick}}(A z)$ is the VTEC estimated by NeQuick model as a function of $A z$ parameter (changing between 0 and $209 \mathrm{sfu}$ ), and $n$ is the number of VTEC values deduced by GIM. 
TABLE 2: Test stations coordinates.

\begin{tabular}{lcccccc}
\hline ID & City & Location & Latitude $($ deg, min, sec) & Longitude $($ deg, min, sec) & Height $(\mathrm{m})$ & MODIP $\left(^{\circ}\right)$ \\
\hline NAIN & Nain & Canada & $56^{\circ} 32^{\prime} 13.07^{\prime \prime} \mathrm{N}$ & $061^{\circ} 41^{\prime} 19.38^{\prime \prime} \mathrm{W}$ & 33.480 & 60.855 \\
PANG & Naples & Italy & $40^{\circ} 49^{\prime} 24.374^{\prime \prime} \mathrm{N}$ & $014^{\circ} 12^{\prime} 58.111^{\prime \prime} \mathrm{E}$ & 122.659 & 48.579 \\
AREQ & Arequipa & Peru & $16^{\circ} 27^{\prime} 55.861^{\prime \prime} \mathrm{S}$ & $71^{\circ} 29^{\prime} 34.068^{\prime \prime} \mathrm{W}$ & 2488.922 & -5.717 \\
\hline
\end{tabular}

Subsequently, on the basis of calculated $A z$ for every monitoring stations, the three coefficients $a_{0}, a_{1}$, and $a_{2}$ are estimated-through a second-order polynomial interpolation-needed to represent the daily ionospheric behaviour in accordance with (7). An example of secondorder polynomial, obtained by fitting the $A z$ parameters computed with Brent method, is shown in Figure 3.

The method adopted to estimate $A z$ parameter is similar to the procedure that the Galileo system will implement [30]; the set of stations used to this purpose has been selected globally distributed on the Earth, to properly study the ionosphere behavior, and is different from the stations belonging to the Galileo ground segment. Moreover, the reference TEC in (9) is obtained from GIMs in this work, while it is derived from dual frequency measurements by Galileo monitoring stations. Hence, the authors assume that the $A z$ values herein estimated are likely near (but not identical) to the ones obtainable by the system in case of full operation $[21,28,30,32]$.

\section{Test and Results}

4.1. Preliminary Analysis. In order to carry out a performance comparison in position domain between NeQuick G and Klobuchar ionospheric corrections, the accuracy analysis of estimated coordinates obtained from a positioning using a single frequency receiver is performed. To evaluate the efficacy of the ionospheric models also the position without ionospheric correction (called "no-iono") is considered. The static single point positioning approach has been conducted in postprocessing, due to the NeQuick coefficients unavailability; the analyzed data are related to the period from 9 to 11 May 2012, using three stations located in different geomagnetic latitudes. The period considered had a low geomagnetic activity; in fact the Average planetary (Ap) index-a daily planetary-scale measure of magnetic activity-achieves low values equal to 26, 10, and 10 for 9, 10, and 11 May 2012, respectively. Table 2 shows the coordinates and the characteristics of the stations considered for the tests.

The utilized data for NAIN and AREQ stations are products available from IGS data centers [28], whereas for PANG station the data are taken from local database of EGNOS Data Collection Network (EDCN).

The used software belongs to a Toolbox developed by PANG (PArthenope Navigation Grouphttp://pang.uniparthenope.it/) except for NeQuick G of ESA property.

The work flow used for position processing is shown in Figure 4. The main inputs are the GPS C1 observables; GPS satellites position at the transmission epoch computed by an orbital propagator starting from broadcast ephemerides [33];

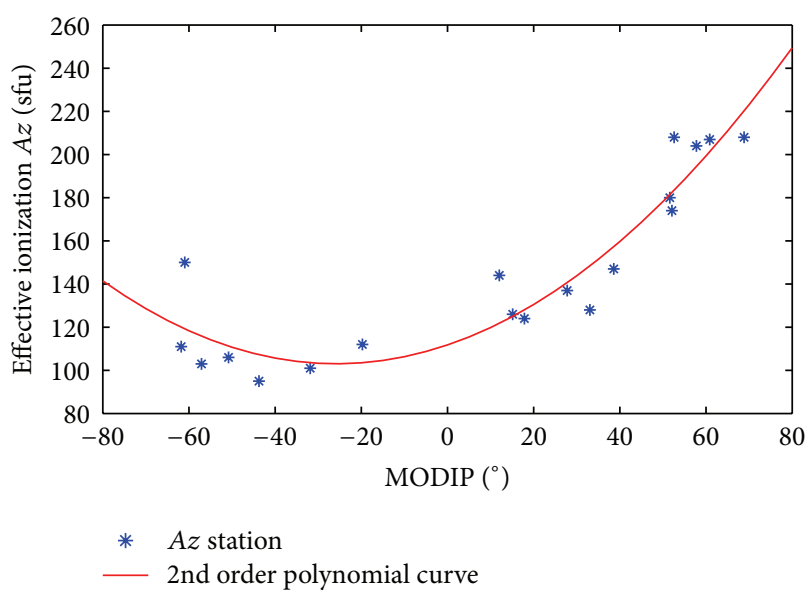

FIGURE 3: Effective ionization second-order optimization.

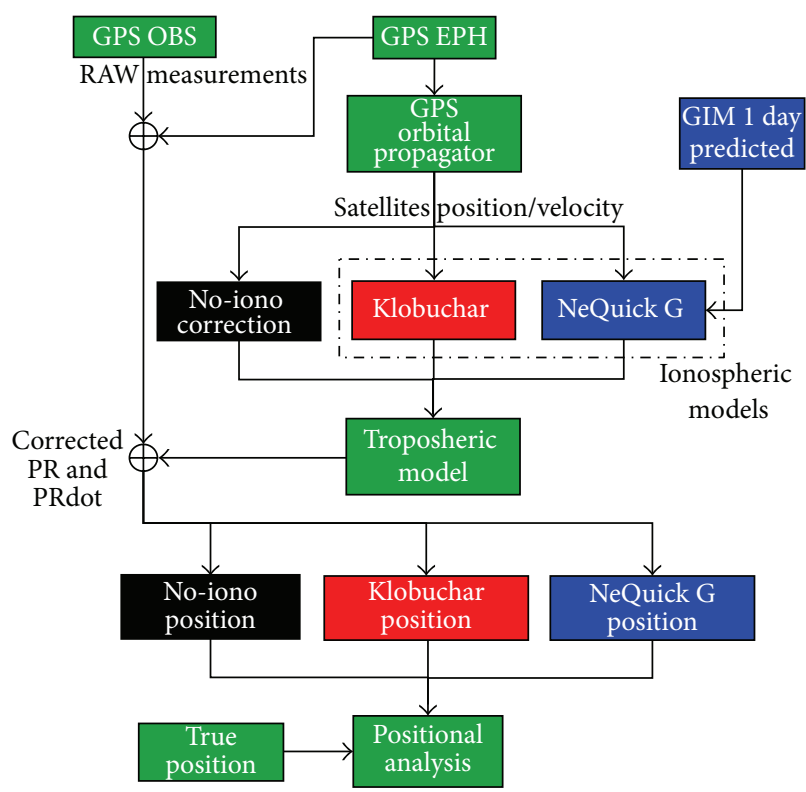

FIGURE 4: Algorithm scheme.

the coordinates of the station; and the predicted GIM (used to estimate the $a_{0}, a_{1}$, and $a_{2}$ coefficients). The error analysis is performed comparing the position obtained using different ionospheric model (i.e., NeQuick G, Klobuchar, and no-iono) with respect to the reference stations coordinates.

Finally in order to analyze the impact of the ionospheric corrections estimated by the different models in position domain, the main statistical parameters (RMS, mean error, 


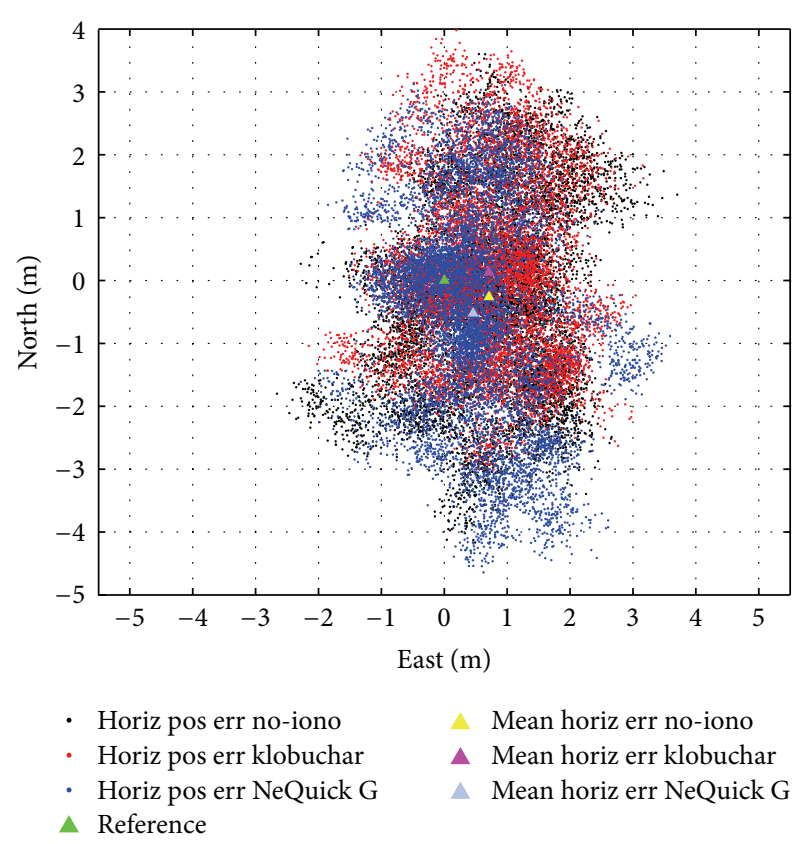

FIGURE 5: Horizontal position error (AREQ station).

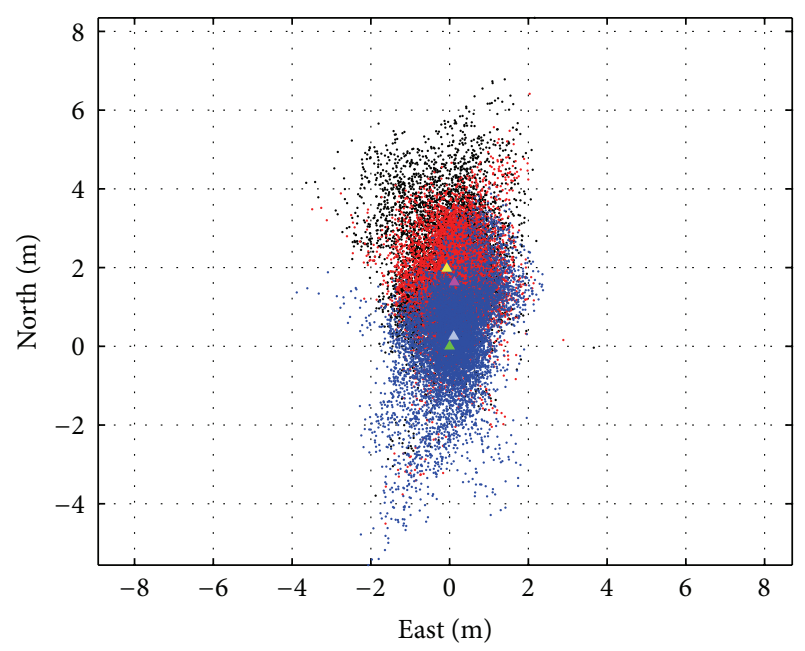

- Horiz pos err no-iono

- Horiz pos err klobuchar

Mean horiz err no-iono

- Horiz pos err NeQuick G

$\Delta$ Reference

FIgURE 6: Horizontal position error (PANG station).

and maximum error) are calculated for both horizontal and vertical components.

4.2. Position Domain Analysis. In this section the positioning results for 3 days data sets, recorded with GNSS receivers, are summarized. Figures 5, 6, and 7 show the horizontal positional error, defined as the difference between the estimate position and the reference one, for all the considered stations (AREQ, PANG, and NAIN) and for all the tested days. The blue points are relative to NeQuick G model, the red ones to

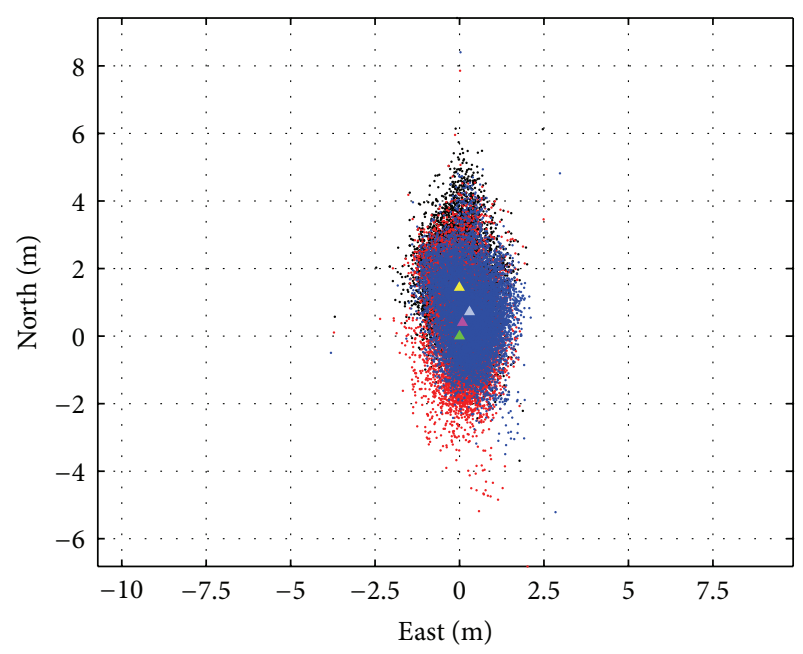

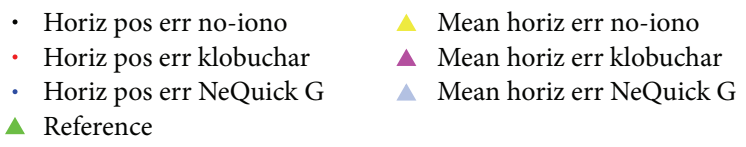

FIGURE 7: Horizontal position error (NAIN station).

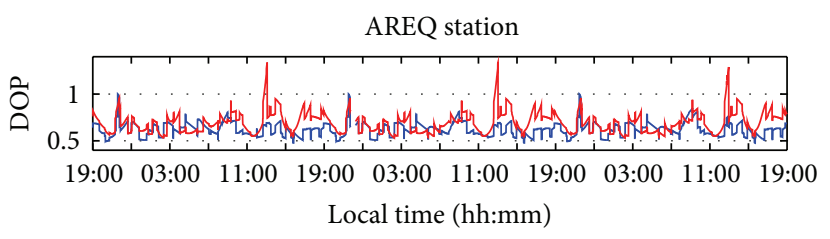

(a)

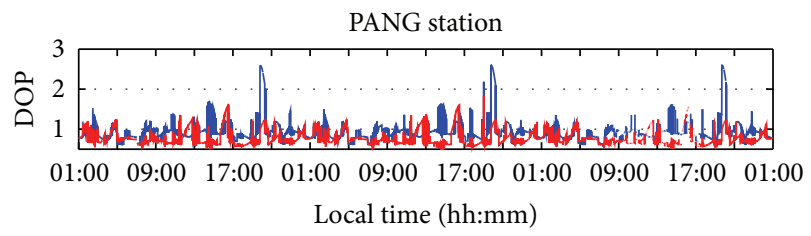

(b)

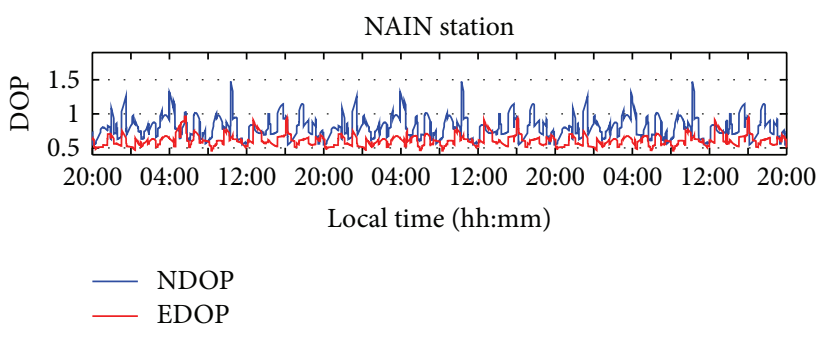

(c)

Figure 8: North and east DOP of all three stations.

Klobuchar, and black points for no-iono configuration. The clouds are very similar, centered on the reference position with a slight improvement using NeQuick G model.

The north and east errors of the AREQ station have a similar value, while the error position along the North-South direction of the PANG and NAIN stations is higher than the error on the East-West direction, due to the satellite geometry, 


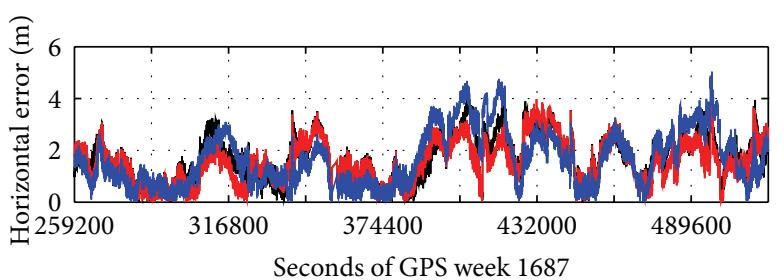

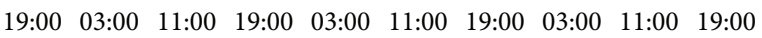
Local time (hh:mm)

(a)

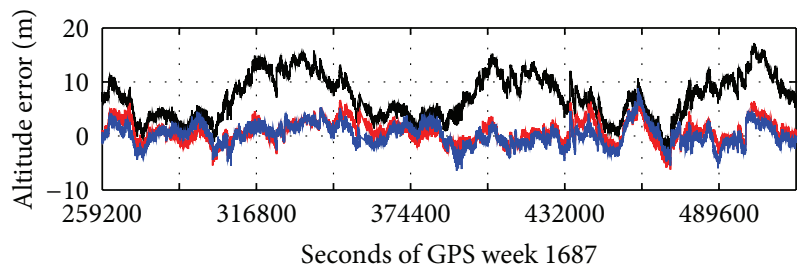

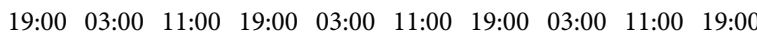

Local time (hh:mm)

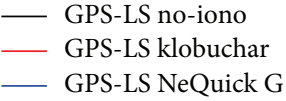

FIGURE 9: Epoch-by-epoch Horizontal and Altitude Errors (AREQ Station).

confirmed by the analysis shown in Figure 8 . In fact, from North Dilution Of Precision (NDOP) and East DOP (EDOP) behavior of the stations under examinations, it emerges that NDOP (blue line) is greater than EDOP (red line) for PANG and NAIN stations while for the equatorial station AREQ the NDOP and EDOP get values generally similar. Specifically for PANG station, the NDOP reaches a maximum value of 2.6 with a mean value of 0.93 , while EDOP reaches a maximum value of 1.82 with a mean value of 0.77 .

In Figures 9, 10, and 11 position errors are plotted versus time (seconds of GPS week and local time); horizontal and vertical errors are shown, respectively, in the upper and lower boxes, for AREQ, PANG, and NAIN stations. The black line symbolizes no-iono corrections, red line represents Klobuchar model, and blue one is relative to NeQuick G.

For the AREQ station the three configurations analyzed are characterized by similar performance in the horizontal component with a slight improvement for the NeQuick G model; the blue line is lower than the others, although the NeQuick $G$ model has a higher maximum error. For the vertical component the effect of the correction provided by the two models is more evident, the red and blue lines are lower than the black one, and the differences between the models are of centimeter order.

For the PANG station the NeQuick G model guarantees improvements with respect to the Klobuchar model in terms of RMS in the horizontal component (passing from 2 to 1.5 meters) and maximum errors for the vertical (from 11.5 to 10 meters) and horizontal (6.7 to 5.9 meters) components. From Figure 10 emerges that the blue line is lower than the red one

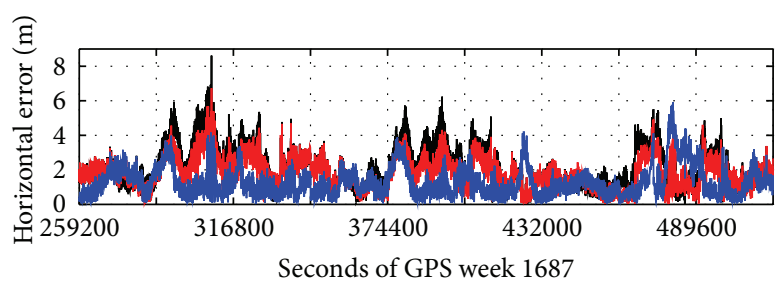

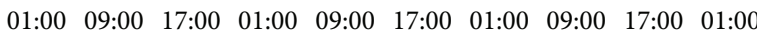
Local time (hh:mm)

(a)

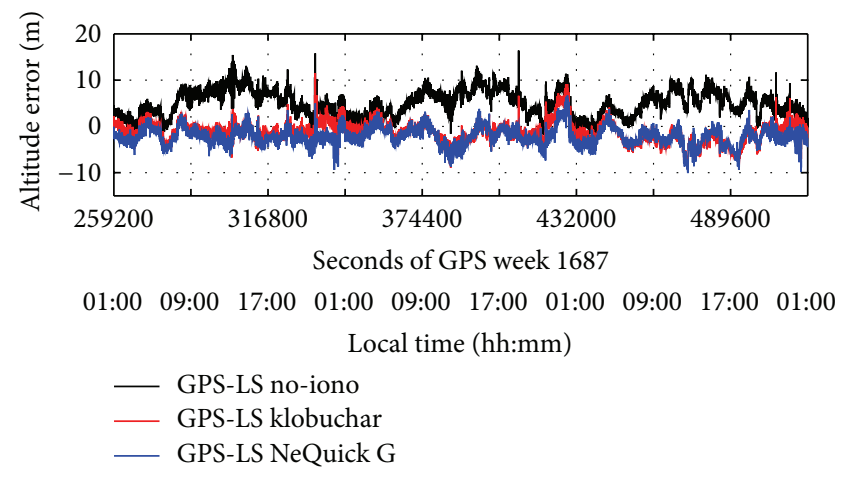

(b)

FIGURE 10: Epoch-by-epoch Horizontal and Altitude Errors (PANG Station).

and the differences between the models are of metric order. The use of the ionospheric models reduces all the considered parameter with respect to no-iono configuration; the blue and red lines are lower of the black one.

For the NAIN station the NeQuick G and Klobuchar models have similar performance, the differences between the models are of submetric order, the use of the ionospheric models reduce the RMS error for both horizontal and vertical component with respect to the no-iono configuration.

From Figures 9 to 11 it can be noted the daily periodic behavior of the horizontal and altitude errors. During the local night time, the error trend reaches its minimum values for both models corrections and for no-iono case and then in the day time all the errors increase (reporting an error peak between the 12:00 and 17:00 local time), more significantly for no-iono correction positioning.

Finally, to summarize the performance of each ionospheric model adopted, in Figure 12 the statistical (RMS, mean error, and maximum error) results for the horizontal and vertical components are shown considering the overall data. The figures of merits of the position errors for all considered days and stations are summarized in Table 3.

Analyzing the final statistical results, showed in Table 3, it emerges that

(i) there is a benefit in positioning if a ionospheric model is used, primarily for the vertical component and for equatorial stations; 
TABLE 3: Summary results.

\begin{tabular}{|c|c|c|c|c|c|c|c|}
\hline Ionospheric model & Station & $\begin{array}{c}\text { Up RMS } \\
(\mathrm{m})\end{array}$ & $\begin{array}{c}\text { Hor RMS } \\
(\mathrm{m})\end{array}$ & $\begin{array}{c}\text { Up mean } \\
\text { Error }(\mathrm{m})\end{array}$ & $\begin{array}{c}\text { Hor mean } \\
\text { Error }(\mathrm{m}) \\
\end{array}$ & $\begin{array}{c}\text { Up max } \\
\text { Error }(\mathrm{m})\end{array}$ & $\begin{array}{l}\text { Hor max } \\
\text { Error }(\mathrm{m})\end{array}$ \\
\hline \multirow{4}{*}{ Without iono correction } & NAIN & 4.317 & 1.934 & 3.812 & 1.438 & 11.234 & 9.417 \\
\hline & PANG & 5.723 & 2.461 & 4.998 & 1.978 & 16.397 & 8.620 \\
\hline & AREQ & 8.3178 & 1.905 & 7.313 & 1.604 & 17.222 & 4.195 \\
\hline & Overall & 6.348 & 2.107 & 5.379 & 1.055 & 17.222 & 9.417 \\
\hline \multirow{4}{*}{ Klobuchar } & NAIN & 1.958 & 1.548 & 1.559 & 0.408 & 10.597 & 7.861 \\
\hline & PANG & 2.436 & 2.053 & 1.900 & 1.634 & 11.542 & 6.731 \\
\hline & AREQ & 2.176 & 1.763 & 1.720 & 0.723 & 7.982 & 3.985 \\
\hline & Overall & 2.193 & 1.793 & 1.722 & 0.764 & 11.542 & 7.861 \\
\hline \multirow{4}{*}{ NeQuick G } & NAIN & 2.057 & 1.510 & 1.597 & 0.774 & 11.234 & 8.305 \\
\hline & PANG & 2.834 & 1.477 & 2.358 & 0.271 & 10.081 & 5.926 \\
\hline & AREQ & 2.065 & 1.974 & 1.668 & 0.694 & 8.869 & 5.035 \\
\hline & Overall & 2.335 & 1.672 & 1.863 & 0.325 & 11.234 & 8.305 \\
\hline
\end{tabular}

TABLE 4: JRC station coordinates.

\begin{tabular}{lcccccc}
\hline ID & City & Location & Latitude $(\mathrm{deg}, \mathrm{min}, \mathrm{sec})$ & Longitude $(\mathrm{deg}, \mathrm{min}, \mathrm{sec})$ & Height $(\mathrm{m})$ & MODIP $\left(^{\circ}\right)$ \\
\hline JRC & Ispra & Italy & $45^{\circ} 48^{\prime} 37.30^{\prime \prime} \mathrm{N}$ & $8^{\circ} 37^{\prime} 47.80^{\prime \prime} \mathrm{W}$ & 279.00 & 52.072 \\
\hline
\end{tabular}

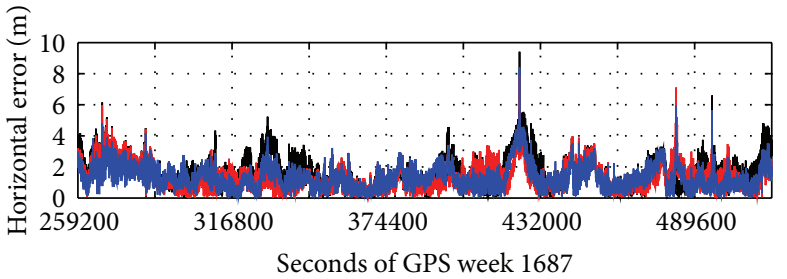

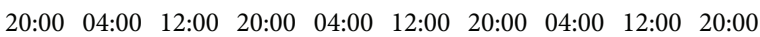
Local time (hh:mm)

(a)

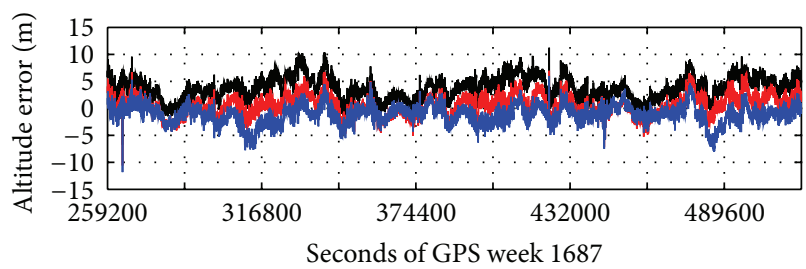

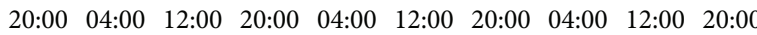
Local time (hh:mm)

- GPS-LS no-iono
GPS-LS klobuchar
GPS-LS NeQuick G

(b)

FIGURE 11: Epoch-by-epoch Horizontal and Altitude Errors (NAIN Station).

(ii) NeQuick G correction guarantees for medium latitude, high latitude and overall stations a more accurate (horizontal RMS) positioning in the horizontal component;

(iii) for the up component NeQuick G correction obtains a better RMS performance only for equatorial station and the best result for overall maximum error.

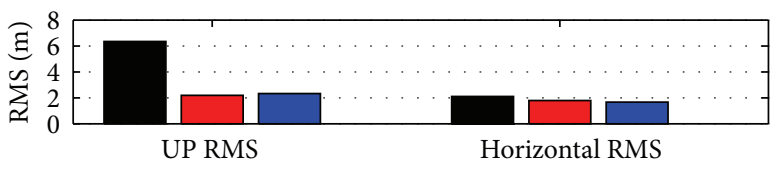

(a)

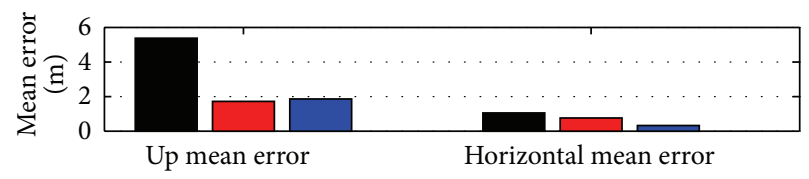

(b)

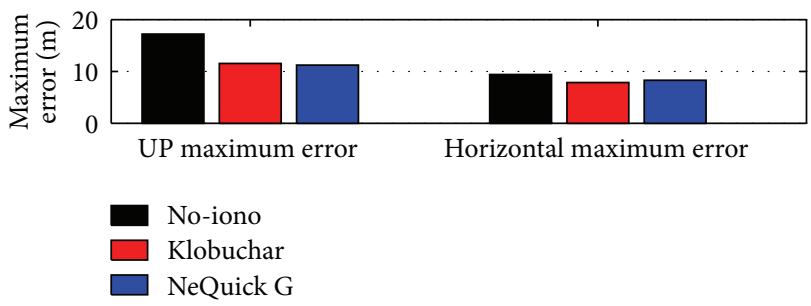

(c)

FIgURE 12: Overall statistical results (all stations).

With NeQuick G a vertical error slightly larger than Klobuchar case is observed for the PANG and NAIN stations, located at middle/high geomagnetic latitudes, while for AREQ station, placed near the geomagnetic equator, a slight vertical improvement relative to Klobuchar is noted. This result shows a worse performance of NeQuick model far away from geomagnetic equator but to definitely demonstrate this concept, analysis of more data relative to a much larger amount of stations placed at different latitudes is required. 
TABLE 5: JRC Summary results.

\begin{tabular}{lcccccc}
\hline Ionospheric model & Station & $\begin{array}{c}\text { Up RMS } \\
(\mathrm{m})\end{array}$ & $\begin{array}{c}\text { Hor RMS } \\
(\mathrm{m})\end{array}$ & $\begin{array}{c}\text { Up mean } \\
\text { Error }(\mathrm{m})\end{array}$ & $\begin{array}{c}\text { Hor mean } \\
\text { Error }(\mathrm{m})\end{array}$ & $\begin{array}{c}\text { Up max } \\
\text { Error }(\mathrm{m})\end{array}$ \\
\hline No-Iono & JRC & 12.31 & 16.58 & 9.14 & 13.19 & 48.05 \\
Klobuchar & JRC & 17.24 & 13.24 & 6.87 & 14.26 & 55.65 \\
NeQuick G & JRC & 18.33 & 13.31 & 6.89 & 15.70 & 54.12 \\
\hline
\end{tabular}

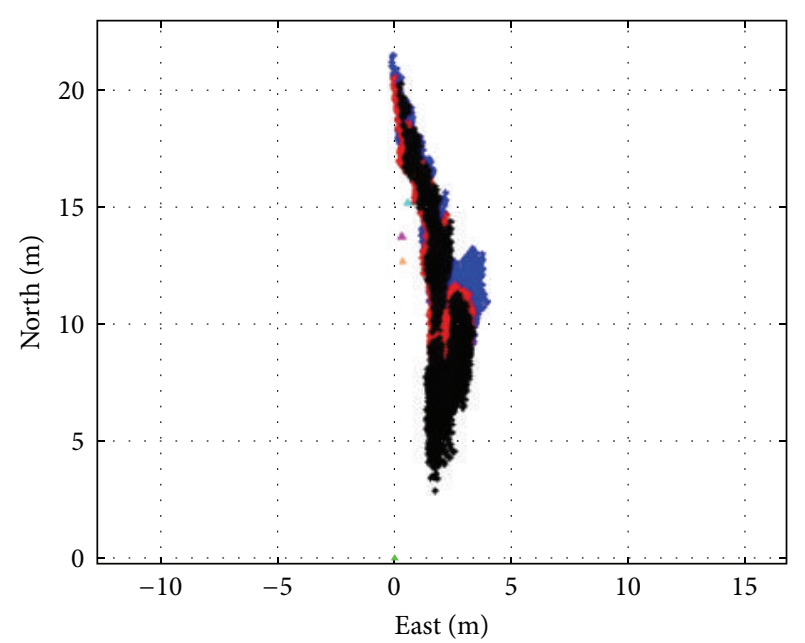

- Horiz pos err NeQuick G

- Horiz pos err klobuchar

- Horiz pos err no-iono

A Mean horiz err NeQuick G

FIGURE 13: Horizontal position error (JRC station).

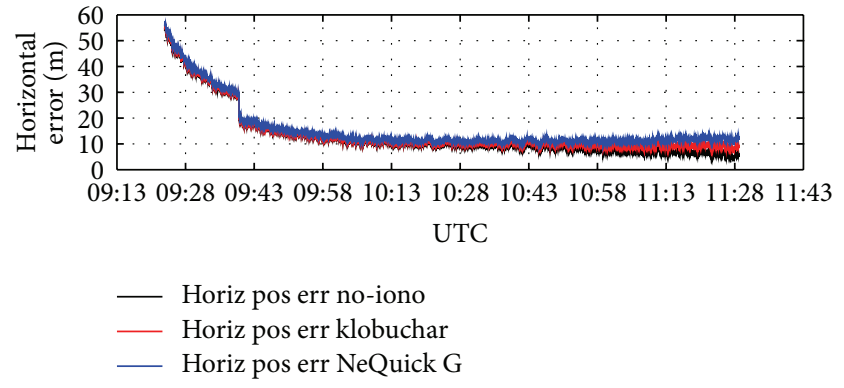

(a)

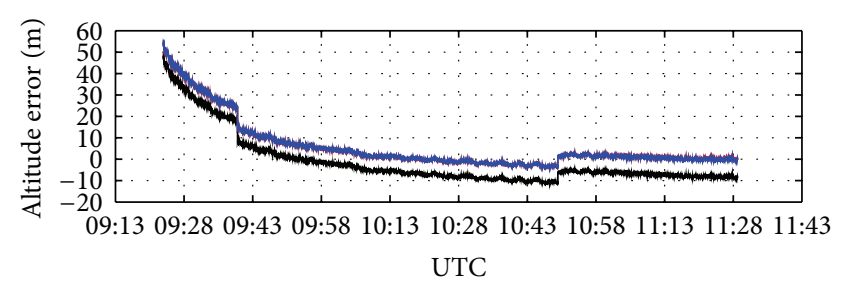

_ Vertical pos err no-iono
_ Vertical pos err klobuchar

(b)

FIGURE 14: Horizontal and altitude errors (JRC station).

In the position domain, using only the Galileo satellites, NeQuick G and Klobuchar models are characterized by a similar performance for all the considered parameters; both models guarantee a slight improvement in the horizontal component with respect to the no-iono configuration, while for the vertical component the models do not enhanced the positioning [34].

\section{Conclusions}

In this study the positioning performance of a single frequency GPS receiver using two ionospheric models, Klobuchar, and NeQuick G, and without ionospheric corrections are statistically analyzed.

In absence of the coefficients to forecast the effective ionization level $A z$ for the NeQuick $G$ model implementation, in this study an alternative approach is adopted to calculate them.

From the results obtained it emerges that NeQuick $G$ and Klobuchar models get comparable performance in position domain. Specifically, although positioning through NeQuick G model achieves up RMS and mean error values slightly greater than Klobuchar ones, in general it offers a
The three models are characterized by similar performance summarized in Table 5.

In Figure 14, position errors are plotted versus time; horizontal error is depicted in the upper box while in the lower one the vertical error is shown. 
better behaviour than Klobuchar model in the horizontal positioning.

The position solution with 10 meters of accuracy is obtained using only Galileo pseudorange measurements as expected; in this case the model analysis is only a preliminary study due to the low number of samples.

\section{Future Work}

The future studies of the authors will be focused on the use of ionization level $A z$ as provided from Galileo navigation message; further investigation using data over longer period of time and from different stations.

\section{Acknowledgments}

The authors wish to acknowledge the European Space Agency/ESA-ESTEC. The software used for part of the work presented in this paper have been provided by the European Space Agency. The views presented in the paper represent solely the opinion of the authors and should be considered as research results not strictly related to Galileo or EGNOS Project design. The authors wish to acknowledge Sandro M. Radicella Head Aeronomy and Radiopropagation ICTP Laboratory (Trieste, Italy) who gave a useful guide. The authors wish to acknowledge the IPSC institute of the JRC for providing Galileo data.

\section{References}

[1] B. Hoffmann-Wellenhof, H. Lichtenegger, and J. Collins, Global Positioning System: Theory and Practice, Springer, Berlin, Germany, 1992.

[2] R. B. Langley, "Propagation of the GPS signals," in GPS for Geodesy, A. Kleusberg and P. J. G. Teunissen, Eds., Springer, Berlin, Germany, 1998.

[3] J. A. Klobuchar, "Ionospheric effects on GPS," in Global Positioning System: Theory and Applications, Vol. I, B. W. Parkinson and J. J. Spilker, Eds., pp. 485-515, American Institute of Aeronautics \& Astronautics, 1996.

[4] J. Hargreaves, The Solar-Terrestrial Environment, Cambridge Atmospheric and Space Science Series, Cambridge University Press, 1992.

[5] A. G. Pavelyev, Y. A. Liou, K. Zhang et al., "Identification and localization of layers in the ionosphere using the eikonal and amplitude of radio occultation signals," Atmospheric Measurement Techniques, vol. 5, no. 1, pp. 1-16, 2012.

[6] A. G. Pavelyev, Y. A. Liou, J. Wickert, K. Zhang, C. S. Wang, and Y. Kuleshov, "Analytical model of electromagnetic waves propagation and location of inclined plasma layers using occultation data," Progress in Electromagnetics Research, vol. 106, pp. 177202, 2010.

[7] C. C. Lee, Y. A. Liou, Y. Otsuka et al., "Nighttime medium-scale traveling ionospheric disturbances detected by network GPS receivers in Taiwan," Journal of Geophysical Research A, vol. 113, no. 12, Article ID A12316, 2008.

[8] A. G. Pavelyev, Y. A. Liou, J. Wickert, T. Schmidt, A. A. Pavelyev, and S. F. Liu, "Effects of the ionosphere and solar activity on radio occultation signals: application to CHAllenging Minisatellite Payload satellite observations," Journal of Geophysical Research A, vol. 112, no. 6, Article ID A06326, 2007.

[9] N. Blaunstein and E. Plohotniuc, Ionosphere and Applied Aspects of Radio Communication and Radar, CRC Press, Taylor \& Francis, 2008.

[10] A. Angrisano, S. Gaglione, C. Gioia, M. Massaro, and U. Robustelli, "Assessment of NeQuick Ionospheric model for Galileo single-frequency users," Acta Geophysicano, vol. 61, no. 6, pp. 1457-1476, 2013.

[11] J. A. Klobuchar, "Ionospheric time-delay algorithm for singlefrequency GPS users," IEEE Transactions on Aerospace and Electronic Systems, vol. 23, no. 3, pp. 325-331, 1987.

[12] G. Di Giovanni and S. M. Radicella, "An analytical model of the electron density profile in the ionosphere," Advances in Space Research, vol. 10, no. 11, pp. 27-30, 1990.

[13] G. Hochegger, B. Nava, S. Radicella, and R. Leitinger, "A family of ionospheric models for different uses," Physics and Chemistry of the Earth, Part C, vol. 25, no. 4, pp. 307-310, 2000.

[14] S. M. Radicella and R. Leitinger, "The evolution of the DGR approach to model electron density profiles," Advances in Space Research, vol. 27, no. 1, pp. 35-40, 2001.

[15] S. M. Radicella and M. L. Zhang, "The improved DGR analytical model of electron density height profile and total electron content in the ionosphere," Annals of Geophysics, vol. 38, no. 1, pp. 35-41, 1995.

[16] A. Angrisano, S. Gaglione, and C. Gioia, "Performance assessment of aided global navigation satellite system for land navigation," IET Radar, Sonar and Navigation, vol. 7, pp. 671-680, 2013.

[17] G. Petit and B. Luzum, "IERS conventions," in IERS Technical Note No. 36, G. Petit and B. Luzum, Eds., pp. 137-150, Verlag des Bundesamts für Kartographie und Geodäsie, Frankfurt, Germany, 2010.

[18] R. B. Bent and S. K. Llewllyn, "Documentation and description of the bent ionospheric model," SAMSO Technical Report 73252, 1973.

[19] R. Leitinger, M.-L. Zhang, and S. M. Radicella, "An improved bottomside for the ionospheric electron density model NeQuick," Annals of Geophysics, vol. 48, no. 3, pp. 525-534, 2005.

[20] B. Arbesser-Rastburg, "The Galileo single frequency ionospheric correction algorithm," in Proceedings of the 3rd European Space Weather Week, Brussels, Belgium, 2006.

[21] B. Nava, P. Coïsson, G. Miró Amarante, F. Azpilicueta, and S. M. Radicella, "A model assisted ionospheric electron density reconstruction method based on vertical TEC data ingestion," Annals of Geophysics, vol. 48, no. 2, pp. 313-320, 2005.

[22] P. Coïsson, S. M. Radicella, R. Leitinger, and B. Nava, "Topside electron density in IRI and NeQuick: features and limitations," Advances in Space Research, vol. 37, no. 5, pp. 937-942, 2006.

[23] S. M. Radiceila, “The NeQuick model genesis, uses and evolution," Annals of Geophysics, vol. 52, no. 3-4, pp. 417-422, 2009.

[24] B. Nava, P. Coïsson, and S. M. Radicella, "A new version of the NeQuick ionosphere electron density model," Journal of Atmospheric and Solar-Terrestrial Physics, vol. 70, no. 15, pp. 1856-1862, 2008.

[25] B. Bidaine, Ionosphere modelling for Galileo single frequency users [Ph.D. thesis], University of Liège, Liège, Belgium, 2012.

[26] F. Azpilicueta, P. Coïsson, B. Nava, C. Brunini, and S. M. Radicella, "Optimized NeQuick ionospheric model for point 
positioning," in Proceedings of International Symposium on GPS/GNSS, pp. 15-18, Tokyo, Japan, November 2003.

[27] SIS-ICD, Galileo Open Service, Signal in Space Interface Control Document, 2006, SISICD-2006. European Space Agency.

[28] A. Aragón-Ángel, R. Orús, M. Hernández-Pajares, J. M. Juan, and J. Sanz, "Preliminary NeQuick assessment for future single frequency users of Galileo," in Proceedings of the 6th Geomatic Week, Barcelona, Spain, 2006.

[29] R. P. Brent, Algorithms for Minimization without Derivatives, Prentice-Hall, Englewood Cliffs, NJ, USA, 1973.

[30] S. M. Radicella, B. Nava, and P. Coïsson, "Ionospheric models for GNSS single frequency range delay corrections," Física de la Tierra, vol. 20, pp. 27-39, 2008.

[31] S. Schaer and W. Gurtner, "IONEX: the IONosphere map exchange, format version 1," in Proceedings of the IGS AC Workshop, Darmstadt, Germany, February 1998.

[32] B. Nava, S. M. Radicella, and F. Azpilicueta, "Data ingestion into NeQuick 2," Radio Science, vol. 46, no. 5, Article ID RS0D17, 2011.

[33] IS-GPS-200, Navstar GPS Space Segment/Navigation User Interfaces, Revision D, ARINC Research, El Segundo, Calif, USA, 2004.

[34] A. Angrisano, S. Gaglione, C. Gioia, D. Borio, and J. FortunyGuasch, "Testing the test satellites: the Galileo IOV measurement accuracy," in Proceedings of ICL-GNSS, Torino, Italy, June 2013. 

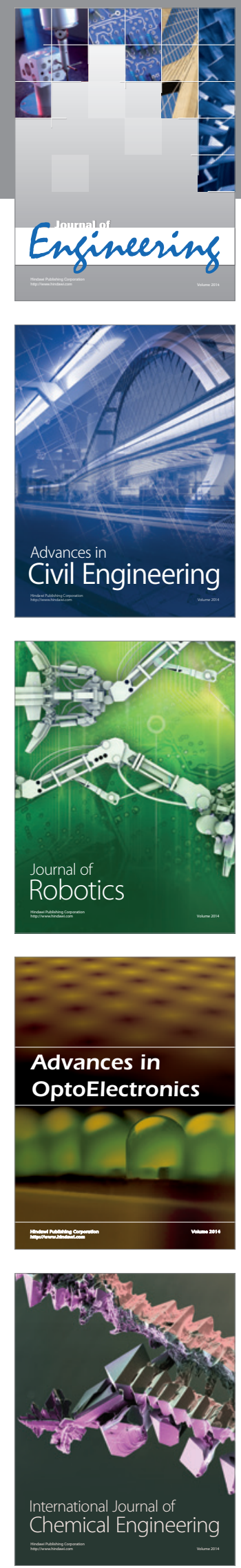

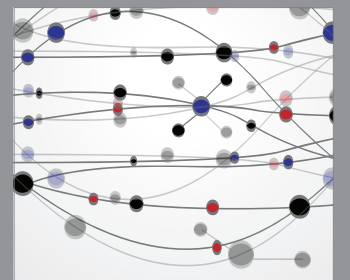

The Scientific World Journal
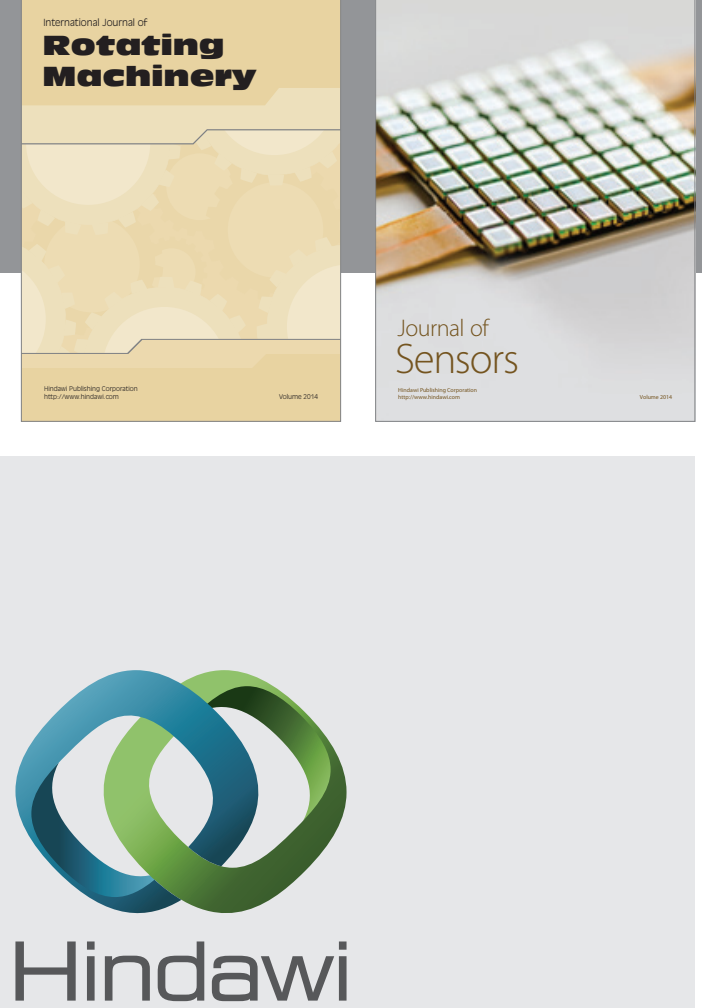

Submit your manuscripts at http://www.hindawi.com
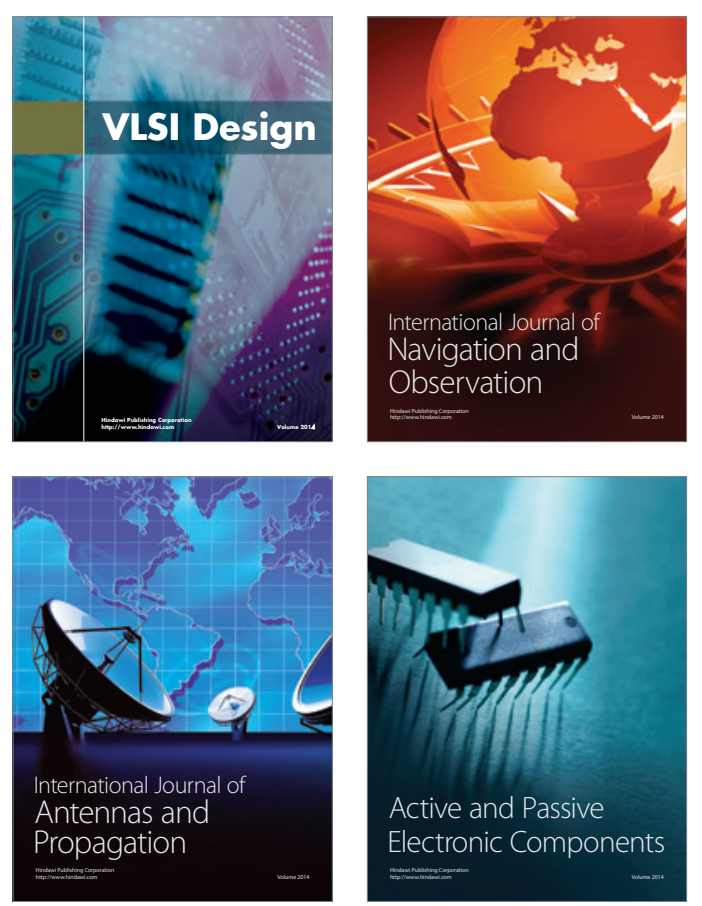
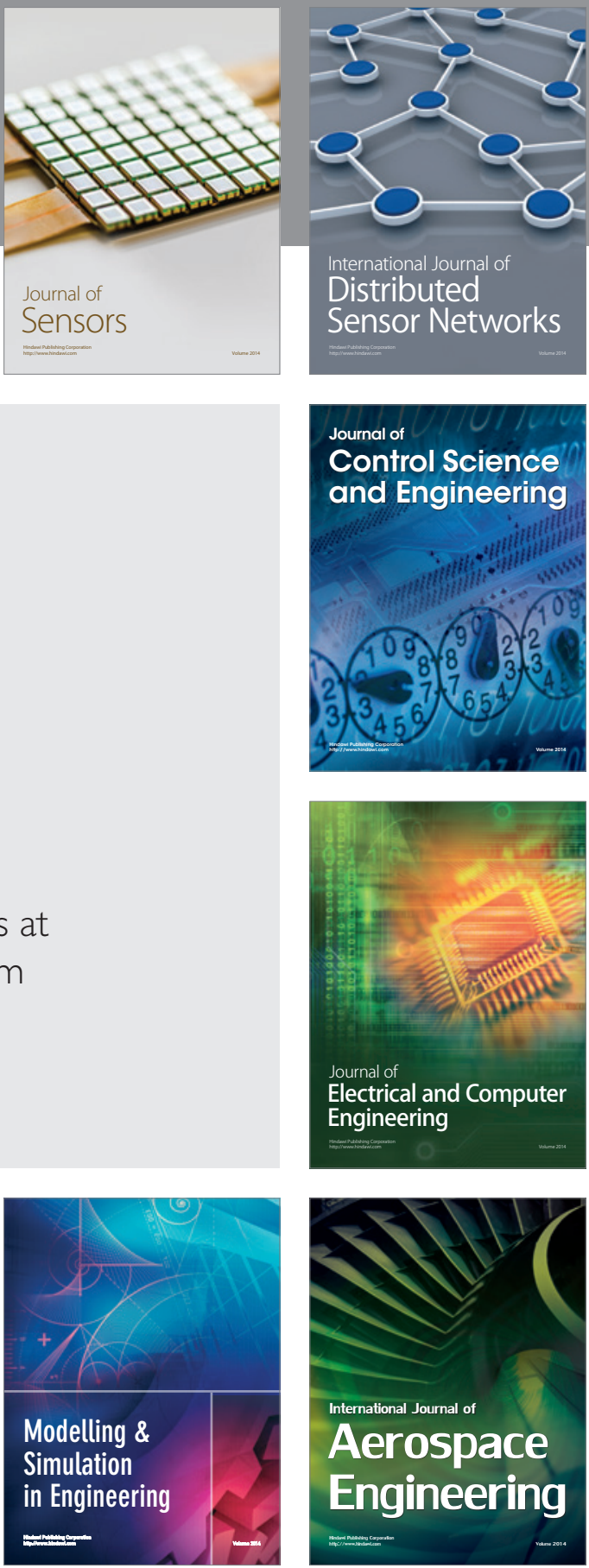

Journal of

Control Science

and Engineering
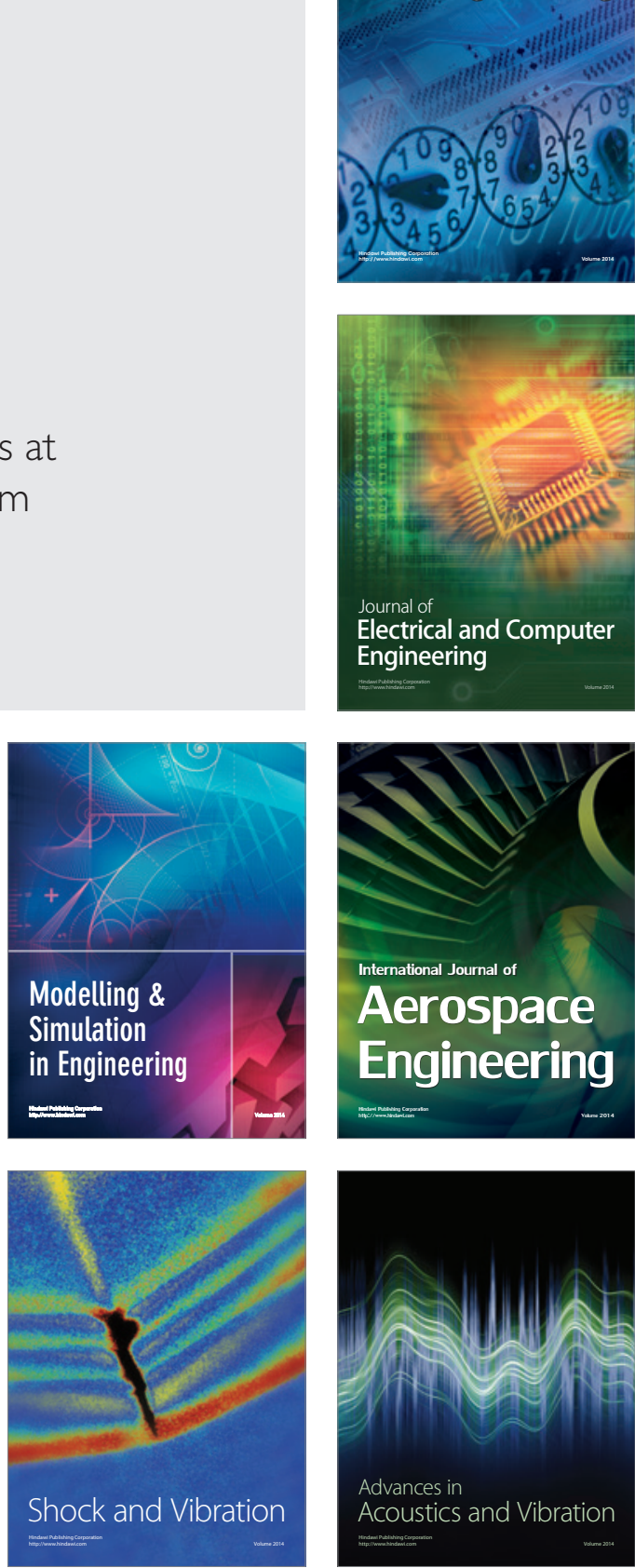